\title{
Introduction to the Special Issue on Ultralow Loss Planar Waveguides and Their Applications
}

W ELCOME to the IEEE JOURNAL OF SELECTED TOPICS IN QUANTUM ELECTRONICS (JSTQE) Special Issue on Ultra Low Loss Planar Waveguides and Their Applications.

Ultra-low loss optical planar waveguide technology is a critical research area driven by the need to improve energy efficiency and advance the power handling capability, performance, function and complexity of photonic integrated circuits and systems-on-chip. An increasing number of applications require lower planar waveguides losses and advances in materials, waveguide design, processing techniques and monolithic and heterogeneous integration. Low loss operation from the visible to the infrared and passive and active waveguide structures as well as linear and non-linear optical devices are needed. Examples of applications driving these advancements include data-communications, bio-sensing, positioning and navigation, low noise microwave synthesizers, spectroscopy, RF signal processing, quantum communication, and atomic clocks.

The special issue comes at an important stage in low loss waveguides and photonic integration and the purpose is to demonstrate the current state-of-the-art in the area of this technology and highlight recent progress and trends to lower waveguide loss, as the key element to realize new functions and their potential applications. This issue brings together 8 invited and 7 contributed papers, authored by world-renowned research groups and scientists, on this topic of ever increasing importance.

The invited papers present significant reviews of state-ofthe art in ultra-low loss waveguide technology and applications and perspectives on future prospects and research directions. Two invited papers cover important history and developments of low loss silicon nitride waveguides, the Photonic Damascene process and the TriPleX process. The remaining invited papers cover important areas of linear and nonlinear optical applications across the visible to mid-infrared, scaling low-loss silicon photonic waveguides to 300-mm wafers for large-scale integration, nonlinear optical frequency micro-combs and their application to RF photonics, low-loss compact lithium niobate electrooptic modulators, trimming low-loss silicon micro-ring resonators for frequency alignment, and polymer waveguides for low loss coupling to silicon waveguides. The contributed papers cover low-loss fabrication techniques using atomic layer deposition (ALD), the use of on-chip Bragg Gratings to measure low optical waveguide losses, device papers on tunable low loss wavelength-flattened directional couplers and highperformance integrated optical resonator circuits and their applications, and large non-linearity Ge-Sb-Se low-loss glass based photonic devices for near-infrared operation. The special issue also includes contributed papers that highlight applications, i.e., low-loss silicon nitride waveguides for optical beamforming networks and continuously tunable optical true time delay using silicon nitride ring resonators.

We hope you will find this JSTQE Issue on Ultra Low Loss Planar Waveguides and Their Applications to be an interesting and useful reference that will impact, stimulate and promote further advances in lowering planar waveguide losses and enable next generations of photonic integration and system-on-chip applications.

\section{ACKNOWLEDGMENT}

We would like to thank the authors of the invited and contributed papers as well as the reviewers who committed their valuable time. This special issue was made possible by the dedicated efforts of the IEEE publications staff and Chin T. Lutz, in particular, for oversight of the special issue. We would like to also thank our previous Journal of Selected Topics in Quantum Electronics Editor-in-Chief, Prof. Luke F. Lester, and our new Editor-in-Chief Prof. José Capmany for their dedication, hard work, and guidance.

\section{J. Blumenthal, Primary Guest Editor University of California, Santa Barbara Santa Barbara, CA 93106 USA danb@ucsb.edu}

\section{Ciminelli, Guest Editor \\ Politecnico di Bari \\ 70126 Bari, Italy \\ caterina.ciminelli@poliba.it}

\section{DAGENAIS, Guest Editor \\ University of Maryland \\ College Park, MD 20742 USA \\ dage@ece.umd.edu}

\author{
B. EGGLETON, Guest Editor \\ University of Sydney \\ Camperdown, NSW 2006, Australia \\ benjamin.eggleton@gmail.com
}

\author{
D. V. Thourhout, Guest Editor \\ Ghent University \\ 9000 Ghent, Bhent, Belgium
}




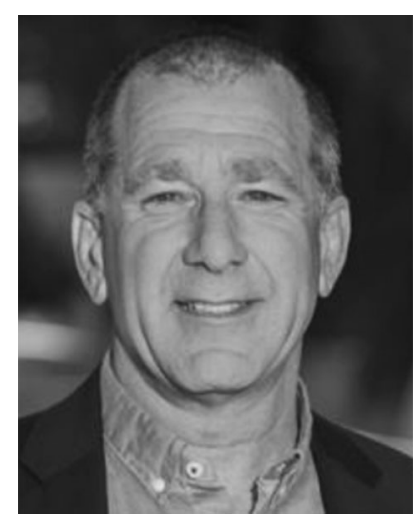

Daniel J. Blumenthal received the B.S.E.E degree from the University of Rochester, Rochester, NY, USA, in 1981, the M.S.E.E. degree from Columbia University, New York, NY, USA, in 1988, and the Ph.D. degree from the University of Colorado Boulder, Boulder, CO, USA, in 1993. He is currently a Professor with the Department of ECE, University of California, Santa Barbara, Santa Barbara, CA, USA, the Director of the Terabit Optical Ethernet Center and heads the Optical Communications and Photonics Integration group. He is Cofounder of Packet Photonics Inc. and Calient Networks, manufacturers of optical communications and network equipment. He holds 22 patents and has authored and coauthored more than 410 papers in the areas of optical communications, optical networks and packet switching, ultra-narrow linewidth and low phase noise integrated lasers, optical gyro sensors, photonic integration in $\mathrm{InP}, \mathrm{SiPh} / \mathrm{InP}$ and ultra-low loss waveguide (ULLW) platforms, and silicon photonic and nanophotonic device technologies. He is coauthor of Tunable Laser Diodes and Related Optical Sources (New York: IEEE-Wiley, 2005) and has invited papers in the PROCEEDINGS OF THE IEEE. He is a Fellow of the National Academy of Inventors, Fellow of the Optical Society of America. He was the Board of Directors for National LambdaRail and as an elected member of the Internet2 Architecture Advisory Council. He is recipient of the Presidential Early Career Award for Scientists and Engineers, a National Science Foundation Young Investigator Award and an Office of Naval Research Young Investigator Program Award and has served on numerous international conference program committees including OFC and Photonics in Switching and as guest editor of several IEEE Journal special issues.

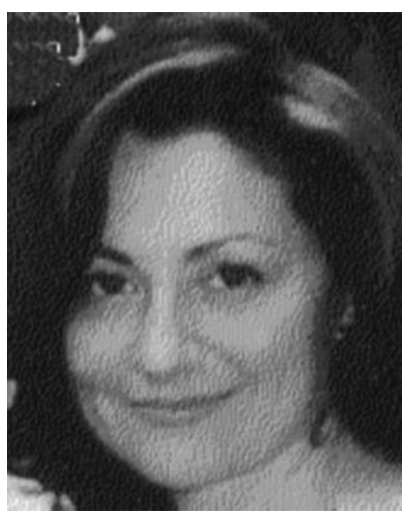

Caterina Ciminelli received the Laurea degree in electronic engineering in 1996 and the Ph.D. degree in electronic engineering in 2000, both from Politecnico di Bari, Bari, Italy. From 1999 to 2002, she has been carrying out industrial research activity on optoelectronic components and subsystems at the R\&D Division of Pirelli Optical Systems, Milan (Italy), and Cisco Photonics Italy, Monza. In May 2002, she joined Politecnico di Bari as an Assistant Professor of electronics. In April 2012, she was appointed as an Associate Professor at Politecnico di Bari. Her research interests include the field of optics and guided-wave optoelectronics, in particular, on modeling, design, fabrication and characterization of devices for optical switching, filtering and sensing, photonic bandgap devices, semiconductor lasers, and optical amplifiers. She has authored and coauthored more than 170 papers. She is a member of OSA. She was a Referee of several international journals. She is involved in several research programs, also as scientific coordinator.

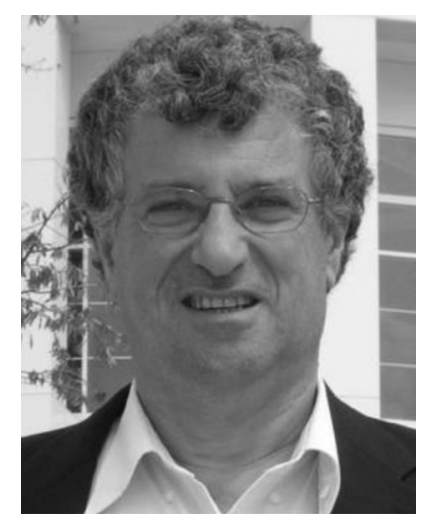

Mario Dagenais (F'10) received the Ph.D. degree in physics from the University of Rochester, Rochester, NY, USA, in 1978, working in quantum optics and photon correlations under the direction of Prof. Leonard Mandel. Together with Jeff Kimble, he made the first observation of photon antibunching. He was a Research Fellow at Harvard University from 1978 to 1980, where he worked in nonlinear optics with Prof. N. Bloembergen. From 1980 to 1987, he worked with GTE Laboratories on photonic switching and semiconductor lasers. In 1987, he joined the University of Maryland, College Park, MD, USA, where he has been a Professor in Electrical and Computer Engineering since 1991. He has more than 300 archival and conference publications. His research interests include $\mathrm{Si} 3 \mathrm{~N} 4 / \mathrm{SiO} 2$ integrated nanophotonic devices on $\mathrm{Si}$, GaN light sources, quantum dot GaAs/AlGaAs, CIGS, and perovskite photovoltaics. He is a Fellow of the Optical Society of America and a Fellow of the Electromagnetic Society. 


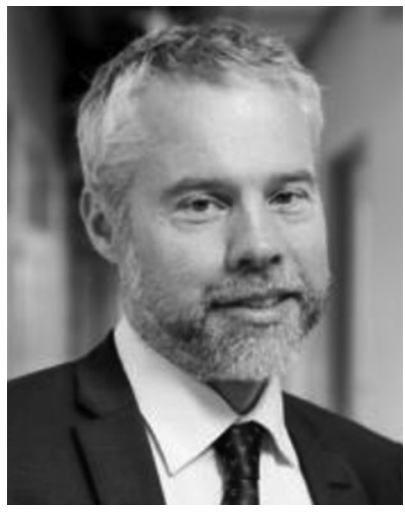

Benjamin Eggleton received the bachelor's degree (hon.) in science and the Ph.D. degree in physics from the University of Sydney, Sydney, Australia, in 1992 and 1996, respectively. He is the Director of the University of Sydney Nano Institute. He is currently a Codirector of the NSW Smart Sensing Network. He was the founding Director of the Institute of Photonics and Optical Science, the University of Sydney from 2009-2018. He was previously an ARC Laureate Fellow and an ARC Federation Fellow twice and was founding Director of the ARC Centre of Excellence for Ultrahigh bandwidth Devices for Optical Systems, from 2003-2017. He then joined Bell Laboratories, Lucent Technologies as a Postdoctoral Member of Staff in the Optical Physics Department under the supervision of Dr. Richard Slusher. In 1998 he transferred to the Optical Fiber Research Department as a Member of Technical Staff and was promoted to Technical Manager of the Fiber Gratings Group in 2000. He was then promoted to Research Director within the Specialty Fiber Business Division of Bell Laboratories, where he was engaged in forward-looking research supporting Lucent Technologies business in optical fiber devices. He has authored and coauthored more than 480 journal publications, including Nature Photonics, Nature Physics, Nature Communications, Physical Review Letters, and Optica and over 200 invited presentations. His journal papers have been cited 19000 times according to Web of Science with an h-number of 66 (86 in google scholar). He is a Fellow of the Australian Academy of Science, the Australian Academy of Technology and Engineering, the Optical Society of America and IEEE. He is Editor-in-Chief of APL Photonics.

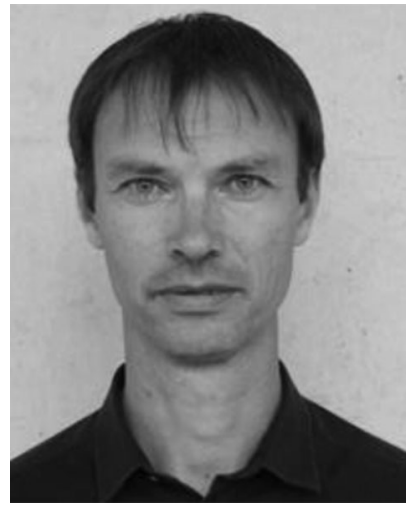

Dries Van Thourhout received the degree in engineering physics and the Ph.D. degree from Ghent University, Ghent, Belgium in 1995 and 2000, respectively. From October 2000 to September 2002, he was with Lucent Technologies, Bell Laboratories, New Jersey, USA. In Oct. 2002, he joined the Department of Information Technology, Ghent University, Belgium, where he is currently a Full Professor. He is also associated to IMEC. His research interest includes the design, fabrication and characterization of integrated photonic devices. Main topics involve silicon nanophotonic devices and the integration of novel materials (III-V, graphene, ferro-electrics, quantum dots, etc.) on these waveguides to expand their functionality. He is working on applications for telecom, datacom, optical interconnect, and sensing. He has authored and coauthored more than 14 patents and 200 journal papers, including several in high ranking journals such as nature photonics, nature nanotechnology, and nanoletters. He has presented invited papers and tutorials on Silicon Photonics at all major conferences in the domain (OFC, ECOC, APC, CLEO). He has coordinated several European Projects (FP6 PICMOS, FP7 WADIMOS, FP7 SMARTFIBER) contributed in many more and is holder of an ERC Grant (ULPPIC). He received the prestigious "Laureaat van de Vlaamse Academie Van Belgie" prize in 2012. 